\title{
I. Ueber die krystallographischen Beziehungen der Methyl- und Aethylsulfinchloroplatinate.
}

\author{
Von
}

\author{
G. J. Laird in Breslau.
}

(Mit 3 Holzschnitten.;

Das Material zu der vorliegenden Arbeit wurde im chemischen Laboratorium der Universität Bonn von den Herren $H$. Klinger und A. Ma as s en dargestellt. Die Genannten beabsichtigten, der Lösung der Frage näher zu treten, ob die vier Affinitäten des Schwefels in den Sulfinverbindungen unter sich gleich oder nicht gleich sind.

Bekanntlich erhält man durch Einwirkung von Jodäthyl $C_{2} H_{5} J$ auf Schwefeläthyl $\left(\mathrm{C}_{2} \mathrm{H}_{5}\right)_{2} \mathrm{~S}$, das Triälbylsulfinjodid, und in analoger Weise auch das Trimethylsulfinjodid $\mathrm{S}\left(\mathrm{CH}_{3}\right)_{3} J$, das Diäthylmethylsulfinjodid $\mathrm{S}\left(\mathrm{C}_{2} \mathrm{H}_{5}\right)_{2} \mathrm{CH}_{3} \mathrm{~J}$, u. s. w. - Durch Schüteln mit feuchtem Ghlorsilber werden die Jodverbindungen in die entsprechenden Chloride ubergeführt. Durch Vermischen der letzteren mit uberschussigem Platinchlorid bilden sich die gut krystallisirenden Aethyl- resp. Methylsulfinchloroplatinate.

Es entsteht nun die Frage, ob beim Ausgange von Jodmethyl und Schwefeläthyl dieselben Verbindungen resp. Platindoppelsalze entstehen, wie beim Ausgange von Jodätbyl und Schwefeläthylmethyl, beziehungsweise ob beim Ausgehen von Jodäthyl und Schwefelmethyl dieselben Verbindungen resultiren, wie von Jodmethyl und Schwefeläthylmethyl.

Eine Arbeit im gleichen Sinne war schon im Jahre 1876 in Kolbe's Laboratorium von Herrn Fr. Krüger*) unternommen worden, und zwar mit dem Resultate, dass die Affinitäten des Schwefels in den Sulfinverbindungen nicht gleich seien, indem Herr $\mathrm{Krüg}$ e r glaubte, aus Aethylsulfid und Jodmethyl ein anderes Diăthylmethylsulfinjodid erhalten zu haben, als wie aus Aethylmethylsulfid und Jodäthyl.

*) "Ueber Isomerien bei organischen Sulfinverbindungen «: : Journ. für prakt. Chem. Neue Folge. 1876, 14, 193-213.

Groth, Zeitschrift f. Krystallogr. XIV. 
Zur Constatirung der Identität oder Verschiedenheit der betreffenden Körper in krystallisirten Derivaten ist nun besonders auch die krystallographische Bestimmung geeignet. Doch mag gleich hier bemerkt werden, dass in der Arbeit des Herrn Krüger die krystallographischen Angaben, welche auch die Verschiedenheit der betreffenden Doppelverbindungen stuttzen sollten, augenscheinlich nur auf äusserlicher Schätzung und nicht auf exacten Messungen beruben und deshalb obne jede Beweiskraft sind.

Vom chemischen Standpunkte aus aber glaubten die Herren Kling er und Ma assen die Arbeit wiederholen zu mussen, weil Herr K r u g e r die Vereinigung von Sulfid und Jodid in der Wärme vor sich gehen liess, und hier die Möglichkeit nicht ausgeschlossen war, dass sich neben einander verschiedene Sulfinjodide bilden konnten. Im Gegensatze bierzu liessen die Herren Kling er und Massen*) die Reactionen zwischen Sulfid und Jodid bei Temperaturen verlaufen, welche $20^{\circ} \mathrm{G}$. nie erreichten. Zum Vergleiche wurden aber dieselben Verbindungen auch nach Herrn K ruger's Methode dargestellt.

Es gelang, eine Reihe von Gold-, Cadmium-, Quecksilber- und Platinverbindungen der Sulfinjodide und Chloride darzustellen, jedoch erwiesen sich nur die Sulfinchloroplatinate als gut messbar. Da aber hiervon die vollständige Substitutionsreihe der Methyl- und Aethylverbindungen vorlag, so war die krystallographische Untersuchung dieser Reihe von grosser Wichtigkeit, und deshalb wurden die betreffenden Salze Herrn Prof. H in $\mathrm{z}$ e zur Untersuchung übergeben. Dieser hatte die Gute, mir die Bearbeitung zu uberlassen, welche in ihren Resultaten nicht nur zur experimentellen Beantwortung der Frage nach der Gleichheit oder Ungleichheit der Affinitäten des Schwefels in den Sulfinverbindungen beiträgt, sondern auch in rein krystallographischer Beziehung, nämlich in Bezug auf die Vicinalflächen, besonders aber auch in Bezug auf die morphotropischen Verhältnisse der Körper neues Material beibringen konnte.

Die Untersuchung erstreckte sich auf die folgenden Verbindungen:

$$
\begin{aligned}
& 2\left[\left(\mathrm{CH}_{3}\right)_{2} \quad \mathrm{~S} . \mathrm{C} \mathrm{H}_{3} \mathrm{Cl}\right]+\mathrm{Pt} \mathrm{Cl}_{4} \\
& \mathbf{2}\left[\left(\mathrm{CH}_{3}\right)_{2} \quad \mathrm{~S} . \mathrm{C}_{2} \mathrm{H}_{5} \mathrm{Cl}\right]+\mathrm{Pt} \mathrm{Cl}_{4} \\
& 2\left[\mathrm{CH}_{3} \cdot \mathrm{C}_{2} \mathrm{H}_{5} \mathrm{~S} . \mathrm{CH} \mathrm{H}_{3} \mathrm{Cl}\right]+\mathrm{PtCl} \\
& \text { 2 }\left[\mathrm{CH}_{3} \cdot \mathrm{C}_{2} \mathrm{H}_{5} \mathrm{~S} \cdot \mathrm{C}_{2} \mathrm{H}_{5} \mathrm{Cl}\right]+\mathrm{PtCl}_{4} \\
& 2\left[\left(\mathrm{C}_{2} \mathrm{H}_{5}\right)_{2} \quad \mathrm{~S} . \mathrm{C} \mathrm{H}_{3} \mathrm{Cl}\right]+\mathrm{PtCl}_{4} \\
& \mathbf{2}\left[\left(\mathrm{C}_{2} \mathrm{H}_{\mathbf{5}}\right)_{2} \quad \mathrm{~S} . \mathrm{C}_{2} \mathrm{H}_{5} \mathrm{Cl}\right]+\mathrm{PtCl}_{4} \text {. }
\end{aligned}
$$

*) Die Arbeit der Herren Klinger und Maassen erscheint gleichzeitig in Liebig's Annalen der Chemie. - Eine vorläufige Mittheilung wurde in der Niederrhein. Ges. für Natur- und Heilkunde zu Bonn in den Sitzungen vom 17. Jan. u. 7. März 1887 gemacht. 


\section{Krystallform des Trimethylsulfinchloroplatinates.}

$$
2\left[\left(\mathrm{CH}_{3}\right)_{3} \mathrm{SCl}\right]+\mathrm{P}_{t} \mathrm{Cl}_{4} \text {. }
$$

Schmelzpunkt: $225^{\circ} \mathrm{C}$.

Krystallsystem: Regulär.

Die kleinen orangerothen (Radde, 4. n) Krystalle stellen die Combination von Oktaëder und Hexaëder dar.

Die Messungen ergaben:

$$
\begin{array}{ccc}
\text { Berechwet: } & \text { Beobachtet: } & \text { Grenzwerthe: } \\
(111):\left(1 T^{\prime}\right)=70^{\circ} 32^{\prime} & 70^{0} 33^{\prime} & 70^{0} 26^{\prime}-70^{\circ} 45^{\prime} \\
(111):(100)=5444 & 5443 & 5437-550
\end{array}
$$

Eine Spaltbarkeit wurde nicht beobachtet. Optisch verhielten sich die Krystalle vollkommen isotrop. - Die durcbsichtigen Krystalle waren kaum über $\frac{1}{2} \mathrm{~mm}$ gross, die grösseren Krystalle aber stets durch schaligen Bau undurchsichtig, so dass die Brechungsindices nicht bestimmt werden konnten.

\section{Krystallform des Dimethyläthylsulfinchloroplatinates.$$
2\left[\left(\mathrm{CH}_{3}\right)_{2} \mathrm{C}_{2} \mathrm{H}_{5} \mathrm{~S} \mathrm{Cl}\right]+\mathrm{PtCl}_{4} \text {. }
$$

Schmelzpunkt: $217^{\circ}-218^{\circ} \mathrm{C}$.

Sowobl die Krystalle der aus Methylsulfid und Jodäthyl dargestellten Chlorverbindung, als die aus Aethylmethylsulfid und Jodmethyl dargestellten sind regulär. Auch blieb es auf die Krystallform ohne jeden Einfluss, ob die Vereinigung von Sulfid und Jodid nach $\mathrm{Kr}$ üger in der Wärme vorgenommen wurde, oder, nach $K \mathrm{l}$ ing e,r und $\mathrm{M}$ a a s s e $\mathrm{n}$, bei Temperaturen unter $20^{\circ} \mathrm{C}$.

Die kleinen orangerothen (Radde, 3. m) Krystalle stellen, wie die

\begin{tabular}{|c|c|c|c|}
\hline$\left(\mathrm{CH}_{3}\right)_{2} \mathrm{SC}_{2} \mathrm{H}_{5} \mathrm{Cl}$ & $\begin{array}{r}\text { Berechnet: } \\
(111):(1 T 1)=70^{\circ} 32^{\prime}\end{array}$ & & $\begin{array}{c}\text { Grenzwerthe: } \\
70^{\circ} 31^{\prime}-70^{0} 33^{\prime}\end{array}$ \\
\hline nach Kr uger & $\{(111):(100)=54 \quad 44$ & $54 \quad 44$ & $54 \quad 43-54 \quad 47$ \\
\hline $\begin{array}{c}\text { dasselbe } \\
\text { nach Kl. und M. }\end{array}$ & $\left\{\begin{array}{l}(111):(1 \overline{1} 1)=7032 \\
(111):(100)=54 \quad 44\end{array}\right.$ & $\begin{array}{l}70.3130^{\prime \prime} \\
5445\end{array}$ & $\begin{array}{lll}70 & 30-70 & 33 \\
54 & 28-55 & 1\end{array}$ \\
\hline $\begin{array}{c}\mathrm{CH}_{3} \mathrm{C}_{2} \mathrm{H}_{5} \mathrm{SCH}_{3} \mathrm{Cl} \\
\text { nach Krü u e r }\end{array}$ & $\left\{\begin{array}{l}(111):(1 T 1)=7032 \\
(111):(100)=5444\end{array}\right.$ & $\begin{array}{ll}70 & 33 \\
54 & 46\end{array}$ & 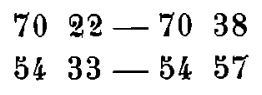 \\
\hline $\begin{array}{c}\text { dasselbe } \\
\text { nach Kl. und M. }\end{array}$ & $\left\{\begin{array}{l}(111):(1 T 1)=7032 \\
(111):(100)=54 \quad 44\end{array}\right.$ & $\begin{array}{ll}70 & 32 \\
54 & 42\end{array}$ & 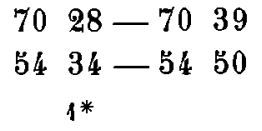 \\
\hline
\end{tabular}
der vorbergehenden Verbindung, Combinationen von Oktaëder und Hexaëder dar.

Winkeltabelle. 
Eine Spaltbarkeit wurde nicht beobachtet. Oplisch verhielten sich die Krystalle völlig isolrop.

An und für sich würde ja natủrlich die Identität der Dimethyläthylverbindung mit der Metbyläthylmethylverbindung nicht durch den Umstand allein bewiesen werden, dass beide regulär krystallisiren. Einerseits jedoch ist, wie wir später sehen werden, die Idenlität des monosymmetrischen Diäthylmethylsulfinchloroplatinates $2\left[\left(\mathrm{C}_{2} \mathrm{H}_{5}\right)_{2} \mathrm{SCH}_{3} \mathrm{Cl}\right]+\mathrm{Pt} \mathrm{Cl}_{4}$ mit dem Aethylmethyläthylsulfinchloroplatinat $2\left[\left(\mathrm{C}_{2} \mathrm{H}_{5} \mathrm{CH}_{3}\right) \mathrm{SC}_{2} \mathrm{H}_{5} \mathrm{Cl}\right]+\mathrm{PtCl}_{1}$ zweifellos, was gewiss auch fur die wirkliche Identität der Dimethylverbindungen spricht; andererseits gelang es aber,'auch in optischer Beziehung die Gleichbeit der beiden letztgenannten regulären Verbindungen zu erweisen.

Aus dem Dimethyläthylsulfinchloroplatinat wurde ein Prisma von $33^{\circ} 48^{\prime}$ brechender Kante geschliffen, welches für die Strablen der Natriumflamme eine Ablenkung von $22051^{\prime}$, also $n=1,6323$ ergab.

Ein Prisma aus der Methyläthylmethylverbindung von 540 32' brechender Kante ergab eine Ablenkung von 42031', also $n=1,6354$.

In Rucksicht darauf, dass bei der Kleinheit der Krystalle die brechenden Kanten nur etwa 1 und $1 \frac{1}{2} \mathrm{~mm}$ lang waren, ist die Uebereinstimmung als eine genugende zu betrachten.

Bemerkenswerth ist, dass die Krystalle des Dimetlıyläthylsulfinchloroplatinates fast sämmtliche Strahlen des Spectrums mit Ausnahme derjenigen in der Gegend des Orange ziemlich vollkommen absorbiren. ISchon die Strahlen der Lithiumflamme gaben ein so lichtschwaches Bild, dass keine brauchbare Einstellung zu erzielen war. Für Grün und Blau sind die Krystalle so gut wie undurchlässig. Mit dem Spectroskop sieht man diese Einfarbigkeit ausgezeichnet markirt.

\section{Krystallform des Diäthylmethylsulfinchloroplatinates.}

$$
2\left[\left(\mathrm{C}_{2} \mathrm{H}_{5}\right)_{2} \mathrm{SCH}_{3} \mathrm{Cl}\right]+\mathrm{PtCl}_{4} .
$$

Schmelzpunkt: $210^{\circ} \mathrm{C}$.

Die Krystalle der Diäthylmetbyl- und der Aethylmethyläthylverbindung sind gleich, ebenso die Krystallisationen verschiedener Darstellungsmethoden.

Krystallsystem : M onosymmetr.isch.

$$
\begin{gathered}
\text { Axenverhältniss: } a: b: c=1,1236: 1: 0,77940 \\
\beta=50^{\circ} 35^{\prime} 20^{\prime \prime} .
\end{gathered}
$$

Beobachtete Formen :

$$
\begin{aligned}
m & =\{110\} \infty P, & c & =\{001\} 0 P, \\
o & =\{111\} P, & b & =\{010\} \infty R \infty .
\end{aligned}
$$


In der umstehenden Winkeltabelle sind gegeben unter der Reihe I die Werthe fül Diäthylmethylsulfinchloroplatinat $2\left[\left(\mathrm{C}_{2} \mathrm{H}_{5}\right)_{2} \mathrm{SCH} \mathrm{CH}_{3} \mathrm{Cl}\right]+\mathrm{PlCl}_{4}$ nach $\mathrm{Kr}$ u g er dargestellt.

Unter II die Werthe für dieselbe Substanz nach Klinger und Ma assen in der Kälte dargestellt.

Unter III die Werthe für Aethylmethyäthylsulfinchloroplatinat $\mathbf{2}\left[\mathrm{C}_{2} \mathrm{H}_{5}\right.$. $\mathrm{CH}_{3} \mathrm{SC}_{2} \mathrm{H}_{5} \mathrm{Cl}$ + $\mathrm{PtCl}_{4}$ nach Kruger, und unter IV die Werthe für diese Substanz nach $K \mathrm{~K}$ inger und $M$ a a s se $n$.

Zu Fundamentalwinkeln wurden die Mittel aller besten Messungen genommen, nämlich :

$$
\begin{aligned}
& m: m=(110):(1 T 0)=81055^{\prime} 30^{\prime \prime} \\
& m: o=(110):(11 \overline{1})=6325 \\
& o: m=(111):(110)=695
\end{aligned}
$$

Hieraus berechnet sich das obige Axenverbältniss und die Winkel :

$$
\begin{aligned}
& m: b \doteq(110):(010)=490 \quad 2 \\
& m: c=(110):(001)=61 \quad 21 \\
& c: o=(001):(111)=5514 \\
& o: b=(T 11):(010)=529 \\
& o: o=(T 11):(\text { TI1) }=75 \quad 42
\end{aligned}
$$

Die umstehende Tabelle beweist wohl zweifellos die Identität aller Krystalle des Diäthylmethylsulfinchloroplatinales verschiedener Herkunft.

Die hyacinthrothen (Radde, 2. $m$ ) Krystalle, unvollkommen spaltbar nach $\{110\} \infty P$, sind meistens so ausgebildet, dass die Combination $\{110\}$ $\{111\}\{001\}$ grosse Aehnlichkeit mit der regulären Combination von Oktaëder und Hexaëder zeigt, vergl. Fig. 1.

Die Symmetrieebene ist nur selten und dann ganz untergeordnet als Krystallfläche ausgebildet.

Zuweilen jedoch erscheinen die Krystalle lang und dünprismatisch durch das Vorherrschen von $\{110\}$ (vergl. Fig. 2), und zwar sowohl die Krystalle von $2\left[\left(\mathrm{C}_{2} \mathrm{H}_{5}\right)_{2} \mathrm{SCH}_{3} \mathrm{Cl}\right]$

Fig. 1.

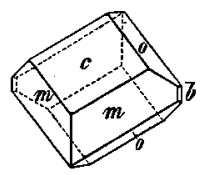

Fig. 2.

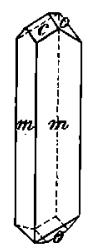
$+\mathrm{Pl} \mathrm{Cl}_{4}$, als auch die von $2\left[\left(\mathrm{C}_{2} \mathrm{H}_{5} \cdot \mathrm{CH}_{3} \mathrm{~S}\right.\right.$ $\left.\mathrm{C}_{2} \mathrm{H}_{5} \mathrm{Cl}\right]+\mathrm{PtCl}_{4}$. Nach gütiger Mittheilung der Herren Klinger und Mass en erscheinen diese letzteren säulenförmigen Krystalle stets aus concentrirt angesetzter Lösung, während anfänglich verdünnte Lösungen die Krystalle des regulären Habitus liefern.

Herr Krüger giebt an, dass das Diäthylmethylsulfinplatinchlorid $2\left[\left(\mathrm{C}_{2} \mathrm{H}_{5}\right)_{2} \mathrm{SCH}_{3} . \mathrm{Cl}\right]+\mathrm{PtCl}_{4}$ ) in regulären Formen krystallisire; namentlich finden sich häufig Würfel, Oktaëder und Tetraëder«. (Es ist schwer, sich 
G. J. Laird.

\begin{tabular}{|c|c|c|c|c|c|c|c|c|c|c|c|c|}
\hline 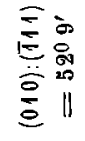 & $\begin{array}{l}\dot{0} \\
\frac{0}{10}\end{array}$ & $\begin{array}{l}0 \\
0 \\
a\end{array}$ & $\begin{array}{l}\text { 足 } \\
\overline{u n} \\
\overline{25}\end{array}$ & | & 1 & 1 & $\begin{array}{l}\text { in } \\
\overline{i n}\end{array}$ & $\stackrel{3}{3}$ & $\begin{array}{l}= \\
=\end{array}$ & 1 & 1 & p \\
\hline 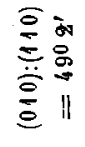 & 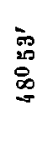 & $\frac{\pi}{g}$ & $\underset{+}{*}$ & I & 1 & I & $\begin{array}{l}\infty \\
20 \\
\infty\end{array}$ & $\stackrel{0}{\infty}$ & 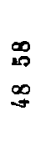 & 1 & 1 & | \\
\hline 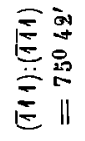 & is & 25 & $\begin{array}{l}\overline{5} \\
20\end{array}$ & 1 & 1 & 1 & 1 & $\begin{array}{l}= \\
\therefore\end{array}$ & I & $\begin{array}{l}\text { 是 } \\
\stackrel{20}{r}\end{array}$ & $\underset{3}{20}$ & $\begin{array}{l}\bar{j} \\
\approx\end{array}$ \\
\hline 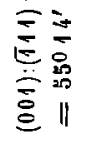 & $\begin{array}{l}\text { in } \\
20 \\
\text { 吾 } \\
20\end{array}$ & $\begin{array}{l}\text { S } \\
\text { 20 } \\
200\end{array}$ & 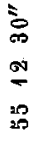 & $\begin{array}{l}\vec{*} \\
\text { से }\end{array}$ & $\begin{array}{l}\infty \\
\infty \\
\infty \\
n \\
n\end{array}$ & $\begin{array}{l}00 \\
\text { oง } \\
20 \\
20\end{array}$ & 1 & 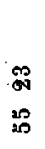 & $\mid$ & 20 & $\begin{array}{l}5 \\
20 \\
20\end{array}$ & $\begin{array}{l}\text { 요 } \\
20 \\
2 \beta\end{array}$ \\
\hline 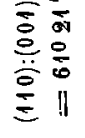 & $\begin{array}{l}\text { जे } \\
\frac{0}{6}\end{array}$ & $\begin{array}{l}\overline{0} \\
\bar{\sigma}\end{array}$ & $\begin{array}{l}\text { बे } \\
\overline{5}\end{array}$ & $\stackrel{+}{\leftarrow}$ & $\begin{array}{l}0 \\
\text { on. } \\
\Sigma\end{array}$ & 엉 & 1 & 1 & 1 & $\frac{0}{6}$ & $\begin{array}{l}\text { 疋 } \\
\bar{\sigma}\end{array}$ & $\begin{array}{l}\text { बे } \\
\overline{6}\end{array}$ \\
\hline 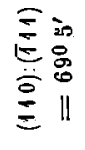 & $\begin{array}{l}\text { in } \\
\text { in } \\
\text { 足 } \\
0\end{array}$ & 암 & $\begin{array}{l}5 \\
0 \\
0 \\
0 \\
8\end{array}$ & I & I & 1 & 1 & 1 & I & $\overrightarrow{0}$ & $\begin{array}{l}- \\
8\end{array}$ & \& \\
\hline 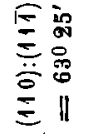 & $\stackrel{\infty}{\infty}$ & $\begin{array}{l}0 \\
\% \\
8 \\
8 \\
8\end{array}$ & \begin{tabular}{l}
$\infty$ \\
\hdashline \\
10
\end{tabular} & 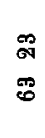 & $\begin{array}{l}5 \\
8 \\
8\end{array}$ & $\begin{array}{l}20 \\
\text { of } \\
0 \\
0\end{array}$ & | & $\begin{array}{l}\text { ON } \\
\text { ते }\end{array}$ & | & $\begin{array}{l}\text { बิ } \\
\text { જี }\end{array}$ & $\begin{array}{l}0 \\
0 \\
80 \\
80\end{array}$ & $\begin{array}{l}\text { ज्ञ } \\
0 \\
0 \\
0\end{array}$ \\
\hline 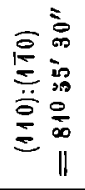 & $\begin{array}{l}\infty \\
\infty \\
0 \\
\infty \\
\infty\end{array}$ & ov & $\begin{array}{l}\infty \\
\infty \\
\infty\end{array}$ & $\begin{array}{l} \pm \\
\bar{\infty}\end{array}$ & $\begin{array}{l}0 \\
\infty \\
\infty\end{array}$ & $\begin{array}{l}\frac{3}{20} \\
\bar{\infty}\end{array}$ & $\begin{array}{l}\bar{\sigma} \\
\bar{\infty}\end{array}$ & $\begin{array}{l}+ \\
\text { ov }\end{array}$ & $\stackrel{\infty}{+\infty}$ & $\begin{array}{l}\bar{\infty} \\
\bar{\infty}\end{array}$ & $\begin{array}{l}\vec{D} \\
\text { 极 }\end{array}$ & $\pi_{20}^{5}$ \\
\hline 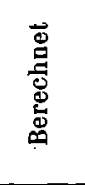 & & & 离 & & & 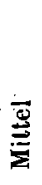 & D. & & 离 & $\frac{8}{8}$ & & 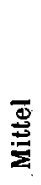 \\
\hline & & - & & & $=$ & & & $\Xi$ & & & $\geq$ & \\
\hline
\end{tabular}


eine richtige Vorstellung dieser Combination zu machen!) Dagegen krystallisirt nach Herrn Krüger das Aethylmethyläthylsulfinplatinchlorid » in langen, wie es scheint, monoklinen Prismen «. Messungen giebt Herr Krüger, wie schon oben erwähnt, nicht an.

Nach den vorhin mitgetheilten Beobachtungen sind ja aber die Angaben des Herrn Krüger leicht zu erklären, denn die Abweichung der monosymmetrischen Krystallform des Diäthylsalzes von der regulären Symmetrie ist nach dem Augenmaasse kaum wahrzunehmen, wie die betreffenden Winkel zeigen :

$$
\begin{array}{ll}
\text { Monosymmetrisch } & (001):(\overline{1} 11)=55014 \\
\text { Regulär } & (001):(111)=34 \quad 44
\end{array}
$$

und

$$
\begin{array}{ll}
\text { Monosymmetrisch } & (110):\left(T_{11}\right)=69 \quad 5 \\
\text { (. Regulär } & (111):(\bar{T} 11)=70 \quad 32 .
\end{array}
$$

Kann auch nach den Messungsresultaten an der Identität der betreffenden Krystalle nicht gezweifelt werden, so zeigen sich doch, wie ein Blick auf die Grenzwerthe in der Vergleichstabelle lehrt, in den Winkeln nicht unerbebliche Schwankungen. Da bei den einzelnen Krystallen diese Schwankungen mit Rücksicht auf die im Allgemeinen gute Oberflächenbeschaffenheit ganz bedeutend über die möglichen Beobachtungsfehler hinausgehen, so müssen sie der Entwickelung von Vicinalflächen zugeschrieben werden. Zwar ist auch an manchen Krystallen die Bildung von Vicinal@ächen durch stumpfe Knickungen beobachtet worden, jedoch war bei weitem häufiger die Verschiebung der Flächen in einheillicher Lage, indem eben trotz einfacher und guter Signalreflexe die Winkelschwankungen sich einstellten.

Es wurde nun versucht, aus den Beobachtungen eine Uebersicht der sich bietenden Erscheinungen zu gewinnen.

Bei genauer Catalogisirung aller Messungsresultate zeigte sich zunächst, dass die Winkelschwankungen durch vicinale Gliederung in gewissen Richtungen viel bäufiger sind, als in anderen. - Als in Richtungen relativ grösster Constanz gelegen, erwiesen sich bei den Krystallen des Diäthylmethylsulfinchloroplatinates die Winkel $(110):(1 \pi 0)$ und $(110):(\pi 11)$. An den zehn besseren der; 20 gemessenen Krystalle wurde die Kante $(110):(1 T 0)$

$$
\begin{gathered}
\text { acht Mal zu } 81056^{\prime} \\
\text { sechs - }-8155 \\
\text { und fün - }-8154 \text { gemessen. }
\end{gathered}
$$

Die Abweichungen an guten Krystallen gingen nicht über $3^{\prime}$ hinaus. 
Die Kante (110): (T11) wurde

$$
\begin{gathered}
\text { fünf Mal zu } 6907^{\prime} \\
\text { drei }-\quad-696 \\
\text { fünf }-\quad-695 \\
\text { vier }-\quad-694 \\
\text { und sieben }-\quad-693 \text { gemessen, während hier, sowie }
\end{gathered}
$$

bei dem letzteren, die Abweichungen selten über 4' hinaus gingen.

Ebenso wurde auch noch eine ziemliche Constanz der Kante (T11):(TTI) beobachtet. Dieselbe wurde

\author{
fünf Mal zu $75^{0} 43^{\prime}$ \\ drei - -7542 \\ und drei - -7541 gemessen. Selten gingen hier die
}

Aloweichungen uber $6^{\prime}$ hinaus.

Am häufigsten war den Schwankungen, auch an gut spiegelnden $\mathrm{Kry}-$ stallen, die Zone (110):(001):(TT1), resp. (1T0):(001):(T11) unterworfen; jedoch ergiebt sich aus den Aufzeichnungen der Messungen, dass besonders die Basis $\{001\}$ in ibrer Lage gegen die constanter bleibenden $\{110\}$ und $\{$ T11\} schwankt. Beispielsweise wurden beobachtet (und zwar beziehen sich selbstverständlich die jeweilig zusammengestellten Winkel auf dieselbe Richtung desselben Krystalles):

$$
\begin{aligned}
& \left.\begin{array}{ll}
(110):(001)=61031^{\prime} & \left(\text { Diff. }+10^{\prime}\right) \\
(001):(\overline{1} 1)=55 & 5 \quad(-\quad-9)
\end{array}\right\}(110):(\overline{1} 1)=116^{0} 36^{\prime}\left(\text { Diff. }+1^{\prime}\right)
\end{aligned}
$$

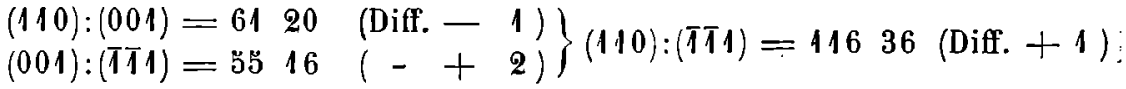

$$
\begin{aligned}
& \left.\left.\begin{array}{l}
(110):(001)=61 \quad 2 \quad(\text { Diff. }-19) \\
(001):(T T 1)=5529 \quad(-+15)
\end{array}\right\}(110):(T T 1)=11631 \text { (Diff. }-4\right)
\end{aligned}
$$

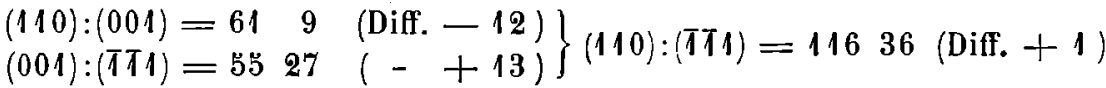

$$
\begin{aligned}
& \left.\left.\begin{array}{ll}
(110):(001)=6124 & (\text { Diff. }+3) \\
(001):(\text { TT1 })=5512 & (-\div 2)
\end{array}\right\}(110):(\text { TT1 })=11636 \text { (Diff. }+1\right) \\
& \left.\begin{array}{l}
(110):(001)=6117 \quad(\text { Diff. }-4) \\
(001):(T T 1)=5520:(-+6)
\end{array}\right\}(110):(\text { TT1) }=11637 \text { (Diff. + } 2 \text { ) } \\
& \left.\begin{array}{l}
(110):(001)=6126 \quad(\text { Diff. }+5) \\
(001):(\bar{T} 1)=558 \quad(-[6)
\end{array}\right\}(110):\left(T^{\top} 1\right)=11634 \quad(\text { Diff. }-1) \\
& \left.\begin{array}{l}
(110):(001)=61 \quad 32 \quad(\text { Diff. }+11) \\
(001):(T T 1)=55 \quad 3 \quad(--11)
\end{array}\right\}(110):(\text { TT1) }=11635 j \text { (Diff. } \quad 0)
\end{aligned}
$$


Ueber die krystallogr. Beziehungen der Methyl- und Aethylsulfinchloroplatinate.

$$
\begin{aligned}
& \left.\begin{array}{l}
(110):(001)=61^{0291^{\prime}}\left(\text { Diff. }+8 \frac{1}{2}\right) \\
(001):(T T 1)=55 \quad 6 \frac{1}{2}\left(-\quad 7 \frac{1}{2}\right)
\end{array}\right\}(110):\left(T_{T}\right)=116^{0} 36^{\prime}\left(\text { Diff. }+1^{\prime}\right) \\
& \left.\begin{array}{l}
(110):(001)=6128 \quad(\text { Diff. }+7) \\
(001):(T T 1)=5510 \quad(-+4)
\end{array}\right\}(110):(T T 1)=11638 \text { (Diff. }+3 \text { ) } \\
& \left.\left.\begin{array}{l}
(110):(001)=61 \quad 18 \quad(\text { Diff. }-3) \\
(001):(T T 1)=5516 \quad(-+2)
\end{array}\right\}(110):\left(T_{T} 1\right)=11634 \quad \text { (Diff. }-1\right)
\end{aligned}
$$

Man sieht hieraus, wie auch bei verhältnissmässig grossen Abweichungen der Winkel (110):(001) und (001):(TT1) der resultirende Winkel $(110):(\bar{T} \bar{T} 1)$ ganz bedeutend geringere Abweichung aufweist.

Was nun die Constanz der Wiederkehr gewisser Vicinalfächen anbelangt, wie sie für den Danburit von Scopi durch Herrn M. Schuster*) und für den Cölestin von Lüneburg durch Herrn C. Hintze ${ }^{* *}$ ) bewiesen wurde, so waren in dieser Beziehung die Verbältnisse an den Krystallen des Diäthylmethylsulfinchloroplatinates weniger günstig, und zwar aus zwei Gründen: Erstens, in den Schwankungen der einheitlichen Begrenzungselemente uberwiegen kleine Intervalle und vermitteln den Uebergang zu den grösseren, wie auch schon die oben mitgetheilte kleine Reibe ersichtlich macht. Die Kleinheit der gewöhnlichen Intervalle zieht aber selbstverständlich eine Unsicherheit nach sich in Bezug auf die richtige Deutung der grösseren Intervalle, als Multipla der kleineren. Zweitens, wie schon vorhin bemerkt, wurde neben der einheillichen Verschiebung der Flächen in vicinale Lage eine Knickung der Flächen in vicinale Begrenzungselemente verhältnissmässig selten beobachtet; und gerade diese vicinalen Knickungen, bei denen die grösseren Intervalle vorzuberrschen pflegen, sind zur Classificirung und Wiedererkennung der Vicinalflächen besonders geeignet. Jedoch in einer Beziehung scheinen die Beobachtungen an den Krystallen dieser Substanz einen neuen und augenscheinlich sehr wichtigen Gesichtspunkt anzudeuten, dass nämlich die Neigungen in den vicinalen Knickungen in einer gewissen Richtung auch für verschiedene Flächen dieselben werden.

Die Knickungen wurden vorzugsweise in der Richtung der Zonen $\{110\}\{11 T\}$ und $\{1 \bar{T} 0\}\{1 T T\}$ beobachtet, und zwar Knickung in zwei F\}ächen, welche zu einander geneigt waren :

$$
\begin{array}{ll}
\text { auf } m \text { um 12, } & \text { auf } 0 \text { um } 11^{\prime} \\
-\quad-\quad-17 & -0-17 \\
-\quad-17 & -o-19
\end{array}
$$

*) „Studien über die Flächenbeschaffenheit und die Bauweise der Danburitkrystalle von Scopi in Graubünden «. Tschermak's min. u. petrogr. Mittheilungen (Neue Folge) 5, 397-455 und 6, 301-514. Referirt in dieser Zeitschr. 11, 277.

**) "Ueber Cölestin von Lüneburg und das Studium von Vicinalflächen a. Diese Zeitschr. 11, 220-235. 


$$
\begin{aligned}
& \text { und nochmals auf } m \text { um } 17 \text { auf } 0 \text { um } 19
\end{aligned}
$$

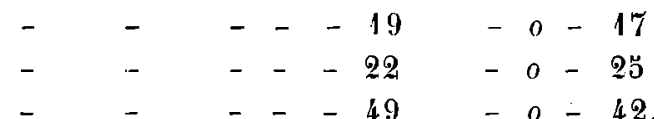

Wenn sich diese Beobachtung, dass gleiche vicinale Knickungen verschiedene Flächen derselben Richtung treffen, auch bei anderem und reichlicherem Beobachtungsmaterial bestätigt, so würden thatsächlich die vicinalen Flächen sich als reine Wachsthumserscheinungen herausstellen, welche durch irgend welche Einflusse bei der Krystallisation hervorgebracht werden, in dem Sinne wie dies schon früher von G. Hintze in seiner citirten Arbeit (S. 233) andeutungsweise ausgesprochen wurde, im Gegensatz zu M. Schuster, welcher fur die Vicinalen auch Gesetzmässigkeiten in den Paramelern in Anspruch nimmt.

Optische Untersuchung. Die Ebene der optischen Axen steht senkrecht zur Symmetrieebene. Die erste Mittellinie steht ungefähr senkrecht zur Verticalaxe, wenig geneigt im Sinne eines positiven Hemidomas.

Die Doppelbrechung ist stark, aber doch nicht so stark wie sonst bei den meisten Platinsalzen; Cbarakter positiv.

Eine Platte senkrecht zur ersten Mittellinie ergab den scheinbaren Axenwinkel in Luft für

$\begin{array}{ccc}\text { die Lithiumflamme } & 330 & 1^{\prime} \\ \text { Natriumflamme } & 31 & \check{2} 2 \\ \text { Thalliumflamme } & 30 & \mathbf{1 3} \\ \text { fur blaues Glas } & 26 & \mathbf{4 6}\end{array}$

Weitel wurde ein Prisma geschliffen, die brechende Kante parallel der Axe der kleinsten Elasticität, die Medianebene parallel den Axen der mittleren und kleinsten Elasticität. Der Winkel der brechenden Kante betrug 270 2'. Das Minimum der Ablenkung für Natriumlicht ergab sich für den parallel der mittleren Elasticitätsaxe schwingenden Strahl $=19^{\circ} 25^{\prime}$, für den parallel der Axe der kleinsten Elasticität schwingenden Strahl $=19^{\circ} 5^{\prime}$,

$$
\text { also } \begin{aligned}
\beta & =1,6872 \\
\gamma & =1,7043 .
\end{aligned}
$$

Die Bilder für Lithium- und Thalliumlicht waren zu lichtschwach, um brauchbare Einstellungen zu geben.

Aus obigem Werthe für $\beta$ und dem scheinbaren Axenwinkel folgt der wahre Axenwinkel fur Natriumlicht:

$$
\begin{aligned}
2 V_{a} & =18^{\circ} 44^{\prime} \\
\text { und ferner } \alpha & =1,6867 .
\end{aligned}
$$


Ueber die krystallogr. Beziehungen der Methyl- und Aethylsulfinchloroplatinate. 11

Der krystallographische Nachweis der Identität der entsprechenden Platinsalze verschiedener Herkunft unterstüzt entschieden die Annahme der Vierwerthigkeit des Schwefels in diesen Substanzen und die Gleichwerthigkeit der Valenzen. Jedoch darf man sich nicht verhehlen, dass bei alledem die Möglichkeit einer molekularen Umlagerung noch immer nicht mit absoluler Sicherheit ausgeschlossen ist.

\section{Krystallform des Triäthylsulfinchloroplatinates.}

$$
2\left[\left(\mathrm{C}_{2} \mathrm{H}_{5}\right)_{3} \mathrm{~S} . \mathrm{Cl}\right]+\mathrm{PlCl} l_{4} \text {. }
$$

Schmelzpunkt: $195^{\circ} \_196^{\circ}$.

Krystallsystem: Monosymmetrisch.

$$
\text { Axenverhältniss: } \begin{aligned}
& a: b: c=1,4930: 1: 1,6504 \\
& \beta=54^{048^{\prime}} .
\end{aligned}
$$

Es wurden beobachtet:

$$
\begin{array}{ll}
a=\{100\} \infty \pm \infty, & r=\{T 01\} P \infty \\
c=\{001\} 0 P, & o=\{111\} P . \\
p=\{110\} \infty P, &
\end{array}
$$

\begin{tabular}{|c|c|c|c|c|}
\hline \multirow[t]{2}{*}{ W in k el } & \multicolumn{3}{|c|}{$\begin{aligned} \text { L a i } & \text { r d } \\
& \quad \text { Beobachtet }\end{aligned}$} & \multirow[t]{2}{*}{$\mathrm{K} \cap 0 \mathrm{P}$} \\
\hline & & Mittel & Grenzwerthe & \\
\hline$p: p=(110):(1 \overline{1} 0)$ & $101022^{\prime}$ & $101^{0} 23^{\prime}$ & $101020^{\prime}-104035^{\prime}$ & - \\
\hline$p: p=(110):(1 / 0)$ & 7838 & $78 \quad 37$ & $7828-7839$ & $78^{\circ} 52^{\prime}$ \\
\hline$p: a=(110):(100)$ & 5041 & $50 \quad 41$ & $5040-504730^{\prime \prime}$ & 5034 \\
\hline$a: c=(100):(001)$ & * & $54 \quad 48$ & $5440-5449$ & $55 \quad 6$ \\
\hline$c: r=(001):(\overline{1} 01)$ & * & $\begin{array}{ll}687 \\
7\end{array}$ & $6755-6812$ & 680 \\
\hline$r: a=(\overline{1} 01):(\overline{1} 00)$ & 575 & $57 \quad \ddot{5}$ & $57 \quad 0-5716$ & - \\
\hline$c: p=(001):(110)$ & $*$ & $68 \quad 37$ & $6830-6842$ & 6840 \\
\hline$p: r=(110):(10 \overline{1})$ & $695130^{\prime \prime}$ & 6952 & $6947-702$ & - \\
\hline$c: o=(001):(\overline{1} 11)$ & $\begin{array}{llll}77 & 23 & 10\end{array}$ & 7724 (арpr.) & - & - \\
\hline$o: 0=(\overline{1} 11):(\overline{1} 11)$ & 10820 & - & 一 & - \\
\hline$o: a=(\overline{1} 11):(\overline{1} 00)$ & 712630 & - & - & 一 \\
\hline $0: r=(\bar{T} \mid 1):(\bar{T} 01)$ & 5410 & $53 \quad$ (appr.) & 一 & - \\
\hline $0: p=(11 T):(110)$ & 340 & 3353 & 一 & - \\
\hline$p: 0=(110):(111)$ & $64 \quad 49$ & - & 一 & 一 \\
\hline
\end{tabular}

Dieses Salz ist schon vor längerer Zeit von Herrn A. Kn o p*) krystallographisch beslimmt worden. Die von Herrn Knop erhaltenen Werthe sind daher in der folgenden Winkeltabelle beigesetzt.

*) Ann. Chem. Phar. Suppl. 4, 91, 1865. 
Die Farbe-der Krystalle des untersuchten Salzes ist carmintoth (Radde, 28. g). Eine ziemlich vollkommene Spaltbarkeit wurde nach $r\{T 01\}$ beobachtet.

Auch hier ist die Ausbildung der Krystalle entsprechend der anfängJichen Concentration der Lösung verschieden. Die besseren Krystalle entstehen aus verdünnt angesetzter Lösung und entsprechen auch im Habitus, wie es scheint, denjenigen, welche Herrn K n op zur Untersuchung vorgelegen haben. Sie sind mehr oder weniger tafelförmig nach der Querfläche $a\{100\}$, häufig gestreckt nach der Symmetrieaxe, und zeigen $c\{001\}$ und

Fig. 3.

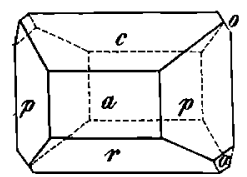
$r\left\{T_{01}\right\}$ meist im Gleichgewicht. (Vergl. Fig. 3.) Die Hemipyramide $o\{(T 11\}$ war nur selten und dann ganz klein ausgebildet.

Die Krystalle aus concentrirl angesetzter Lösung sind langprismatisch nach der Verticale, und zwar entweder nadelförmig bis spiessig, oder auch dünnblätterig durch Vorherrschen von zwei parallelen Prismenflächen.

An den Krystallen dieser dünnblätlerigen Ausbildung wurden auch Zwillinge beobachtet. Die Messungen ergaben als Zwillingebene $z\{\overline{6} 35\} \frac{6}{5} p 2$, wie sich aus folgenden Winkeln ergiebt.

$$
\begin{aligned}
& \text { Berechnet: } \\
& p: \underline{p}=(\bar{T} \bar{T} 0):(\underline{T 10})=1102 \tau^{\prime} \\
& p: \underline{p}=(\bar{T} 10):(\underline{T} 0)=10446 \\
& \text { Beobachtet: } \\
& 120 \text { (approx.) } \\
& 10530^{\prime} \text { (approx.) }
\end{aligned}
$$

Die Zwillinge sind so ausgebildet, dass als Verwachsungsfliache die linke untere Fläche $(6 \overline{3} \overline{5})$ des rechten Individuums fungirt, also natürlich die rechte untere (63) $)$ des linken Individuums.

Die Flächenschwankungen an guten Krystallen sind so gering, dass hier von dem Eingehen auf Vicinalflächen keine Rede sein konnte. Vicinale Knickungen wurden nur ganz vereinzelt beobachtet.

Opt ische Untersuchung. Die Ebene der optischen Axen ist senkrecht zur Symmetrieebene, ungefähr parallel $r\{T 01\}$, aber weniger als diese gegen die Verticale geneigt.

Die Symmetrieaxe ist die zweite Mittellinie.

Doppelbrechung stark und negativ.

Eine Platte senkrecht zu der in der Symmetrieebene liegenden Mittellinie geschliffen ergab:

$$
\begin{aligned}
& 2 H_{a} \text { Lithiumflamme }=101047^{\prime} \\
& 2 H_{a} \text { Natriumflamme }=103 \quad 48 \\
& 2 H_{o} \text { Thalliumflamme }=104 \quad 41
\end{aligned}
$$

Eine Platte parallel der Symmetrieebene ergab: 
Ueber die krystallogr. Beziehungen der Me(hyl- und Aelhylsulfinchloroplatinate. 13

$$
\begin{aligned}
& 2 H_{0} \text { Lithiumflamme }=105^{0} 39^{\prime} \\
& 2 H_{0} \text { Natriumflamme }=10426 \\
& 2 H_{a} \text { Thalliumflamme }=103 \quad 16
\end{aligned}
$$

Man siebt, dass die erste Mittellinie zwar dieselbe für die rothen und gelben Strahlen, aber alsdann zweite Mittellinie für die grünen Strahlen ist.

Aus obigen Werthen folgt:

$$
\begin{aligned}
& 2 V_{a} \text { fur Lithium } 88^{\circ} 29^{\prime} \\
& 2 V_{a}-\text { Natrium } 89 \quad 4.520^{\prime \prime} .
\end{aligned}
$$

Der an der ersten Platte für Thalliumlicht gemessene Winkel war nicht so zuver]ässig als die übrigen bestimmt und wurde deshalb nicht zur Berechnung von $2 V_{a}$ für $T l$-Licht verwerthet.

Da die Brechungsindices des angewandten Oeles waren:

$$
\begin{aligned}
& n \text { Lithiumflamme }=1,4647 \\
& n \text { Natriumflamme }=1,4678 \\
& n \text { Thalliumflamme }=1,4708,
\end{aligned}
$$

so ergab sich aus den Werthen von $H_{a}$ und $V_{a}$ für $L i$ - und $N a$-Licht der mittlere Brechungsindex der Krystalle

$$
\begin{aligned}
& \beta \text { für Lithiumflamme }=1,6290 \\
& \beta-\text { Natriumflamme }=1,6371 .
\end{aligned}
$$

Weiter liefert der Cauch y'sche Interpolationsausdruck

$$
\beta \text { für Thalliumflamme }=1,6446 \text {. }
$$

Hieraus und dem Werthe für $2 H_{a}$ folgt:

$$
\begin{aligned}
2 V_{a} \text { Thalliumflamme } & =8903^{\prime} \\
\text { und } 2 H_{0}- & =1054320^{\prime \prime} .
\end{aligned}
$$

In Luft können die optischen Axen auch nicht mehr im spitzen Winkel austreten.

Interessant ist die Vergleichung der Krystallform des Triäthylsulfinchloroplatinates mit der des Triäthylselenchloroplatinates $2\left[\left(\mathrm{C}_{2} \mathrm{H}_{5}\right)_{3} \mathrm{Se} \mathrm{Cl}\right]$ $+\mathrm{PtCl}_{4}$, welche von Herrn W. Schimper*) an der von Herrn L. v. Pieverling**) dargestellten Substanz bestimmt wurde. Nach den Angaben des Herrn Schimper ist Flächencombination und Habitus der Krystalle des Selensalzes ganz analog wie beim Sulfinsalz. Die monosymmetrischen Krystalle des Selensalzes stellen Combinationen von $\{100\}\{001\}$ $\{101\}\{110\}$ dar. Die Form $\{111\}$ wird nicht angegeben.

*) Diese Zeitschr. 1, 218.

**) Ber. d. d. chem. Gesellschaft 1876, 1, 1471. 
Die entsprechenden Winkel beim Selen- und Sulfinsalz sind:

\begin{tabular}{|c|c|c|}
\hline \multicolumn{2}{|c|}{ Selensalz: } & \multirow{2}{*}{$\begin{array}{c}\text { Sulfinsalz: } \\
54^{0} 48^{\prime}\end{array}$} \\
\hline$a: c=(100):(001)=$ & $58^{\circ} \quad 6^{\prime}$ & \\
\hline$c: r=(001):(\pi 01)=$ & 620 & 687 \\
\hline$r: a=(10 \pi):(100)=$ & 5954 & 575 \\
\hline$c: p=(001):(110)=$ & $73 \quad 48$ & 6837 \\
\hline$p: r=(110):(10 \pi)=$ & 740 & $695130^{\prime \prime}$ \\
\hline$p: p=(1 \mid 0):\left(1 T_{0} 0\right)=1$ & 1138 & 10122 \\
\hline
\end{tabular}

Die Axenverhältnisse sind bei obiger Stellung:

$\begin{array}{ccccccc} & a & b & c & \beta & a: c \\ \text { Selensalz } & 1,7893: 1: 1,8261 ; & 58^{0} 6^{\prime} ; & 1: 1,0206 \\ \text { Sulfinsalz } & 1,4930: 1: 1,6504 ; & 5448 ; & 1: 1,1054 .\end{array}$

Man könnte natürlich auch die Stellung der Krystalle so vertauschen, dass $c\{001\}$ des Selensalzes zu $r\{104\}$ genommen würde, dann wäre zwar der entsprechende Winkel $a\{100\}: r\{\overline{101}\}$ beim Selensalz $=58^{0} 6^{\prime}$ und beim Sulfinsalz $=57^{\circ} 5^{\prime}$, jedoch würde dann für die anderen Winkel $a(100): c(001)$ und $c(001): r(\bar{T} 01)$ eine viel geringere Uebereinstimmung resultiren, während freilich auch dann die beobachteten Spaltungsrichtungen parallel gingen. Die Summe der Differenzen bleibl am.kleinsten bei obiger Vergleichsaufstellung.

Trotz der unzweifelhaften krystallographischen Analogie des Selenund Sulfinsalzes würde doch kaum noch die Annahme einer Isomorphie im strengen alten Sinne zulässig sein, wäbrend bei der von Herrn C. Hintze*) vorgeschlagenen Betrachtungsweise in der verhältnissmässig grossen $\mathrm{Ab}$ weichung der Krystallformen eben die morphotropische Kraft des den Schwefel ersetzenden Selens ihren Ausdruck findet.

Was nun weiter die morphotropischen Beziehungen der Methyl- und Aethylsulfinchloroplatinate unter einander anlangt, so krystallisiren also das Trimetbyl- und Dimethyläthylsulfinchloroplatinat regulär. Die Substitution einer Methylgruppe durch eine Aethylgruppe war also nicht irn Stande, die Gleichgewichtslage der regulären Symmetrie in eine andere von niederer Symmetrie uberzufuhren. Bleibt aber überhaupt der Grad der Symmetrie unverändert, so kann ja natürlich innerhalb der regulären Symmetrie keine weitere Aenderung eintreten. Dagegen sehen wir in der Krystallform des Diälhylmelhylsulfinchloroplatinates, dass der Eintritt einer weiteren Aethylgruppe die Symmetrie der Gleichgewichtslage in revolutionärer Weise verringert hat. Das Diäthylmethylsulfinchloroplatinat krystallisirt monosymmetrisch; doch auch in dieser Gleichgewichtslage weist der

*) „Beziehungen zwischen Krystallform und chem. Constitution «. Verhandl. d. Naturh. Vereins der preuss. Rheinlande und Westfalens. Jahrg. XXXXI. 5. Folge. 1, 261-277. (Referirt in dieser Zeitschr, 11, 158.) 
Habitus der Krystalle und weisen die Flächenwinkel eine so grosse Aehnlichkeit mit der regulären Krystallform des Trimethyl- und Dimethyläthylsalzes auf, dass nach blossem Augenschein (wie die falsche Bestimmung des Herrn Krüger beweist) die monosymmetrischen Krystalle noch für regulär gehalten werden können. Die analogen Winkel sind oben bereits verglichen worden. Die Symmetrieebene der monosymmetrischen Diäthylmethylsulfinchloroplatinatkrystalle entspricht einer Hauptsymmetrieebene der regulären Krystalle.

In der Krystallform des Triälhylplatinsalzes sehen wir, dass die vollständige Ersetzung der Metbyl- durch die Aethylgruppen den Symmetriegrad der Krystalle des Diäthylsalzes nicht weiter verringert hat. Auch das Triäthylsalı krystallisirt monosymmetrisch. Sowohl der krystallonomische Habitus, als die Axenverhältnisse der Krystalle des Triäthyl- und des Diäthylmethylsulfinchloroplatinates weichen aber bei ungezwungener Aufstellung beträchtlich von einander ab. Die Kanten der Prismen- und Pyramidenflächen stimmen bei homologer Aufstellung der Krystalle mit parallelen Symmetrieebenen nicht entfernt tiberein. Dennoch aber zeigen die monosymmetrischen Krystalle des Triähylsalzes sowohl im Habitus, als auch in entsprechenden Winkeln wieder eine unverkennbare Aehnlichkeit mit den Krystallen der regulären Salze. Bei unserer Aufstellung bilden die Flächen (001) (T01) (110) das pseudoreguläre Oktaëder, während die Querfläche (100) der Wưrfelfläche entspricht.

Die analogen Winkel sind:

$$
\begin{aligned}
& \text { Regulär } \quad(001):(111)=54^{0} 44^{\prime} \\
& \text { Monosymmetrisch }\left\{\begin{array}{lr}
(100):(001)=54 & 48 \\
(100):(101)=57 & \text { a } \\
(100):(110)=50 & 41
\end{array}\right. \\
& \text { Regulär } \quad(111):(\overline{1} 11)=7032 \\
& \text { Monosymmetrisch }\left\{\begin{array}{l}
(110):(10 T)=695130^{\prime \prime} \\
(001):(110)=6837
\end{array}\right.
\end{aligned}
$$

und zwar entspricht hierbei die Symmetrieebene der monosymmetrischen Krystalle des Triäthylsalzes nicht einer Haupisymmetrieebene, sondern einer gewöhnlichen Symmetrieebene (Dodekaëderfläche) der regulären Krystalle. Dies erklärt auch die Verschiedenheit der Triäthyl- und Diäthylmethylkrystalle in krystallonomisch homologer Stellung. Wir bekommen die Winkelanalogie zwischen beiden erst dann, wenn wir die Krystalle in der pseudoregulären Aufstellung mit einander vergleichen.

Diäthylmethyl :

$$
\begin{aligned}
& (001):(111)=55014^{\prime} \\
& (110):(\overline{1} / 1)=69 \quad 5 \\
& (1 \bar{T} 0):(\bar{T} T)=69 \quad 5 \\
& \text { Triäthyl : } \\
& 54^{0} 48^{\prime}=(100):(001) \\
& 6837=(001):(110) \\
& 695130^{\prime \prime}=(110):(10 \overline{1}) \text {. }
\end{aligned}
$$


16 G. J. Laird. Ueb. d. krystall. Bezieh. der Methyl- u. Aethyylsulfinchloroplatinate.

Diese Verhältnisse weisen darauf hin, dass hier bei der Aenderung der Gleichgewichtslage durch Substitutionen im Molekül die krystallographisch ausgezeichneten Richtungen parallel oder senkrecht zur Symmetrieaxe vor anderen Richtungen keinen Vorzug in Rucksicht auf Constanz besitzen, dass es mehr Krystallhabitus und die hierdurch bedingten reingeometrischen Richtungen sind, welche bei der Umlagerungineine andere Gleichgewichtslage maassgebend bleiben, und dass für die Vergleichung der Krystallformenzur Erkenntniss morphotropischer Beziehungen keineswegs nur die Stellungen mit homologer Symmetrie jene Erkenntniss zu befördern geeignet sind.

Vorstehende Untersuchungen wurden im mineralogischen Institut der Universität Breslau, unter Leitung des Herrn Prof. Dr. Hin tze ausgefuhrt. Ich bin dem Letzteren für seine wohlwollenden Rathschläge sehr verpflichtet, und freue mich, demselben an dieser Stelle meinen lebhaftesten und herzlichsten Dank aussprechen zu können. 